Evangeline Wassmer, $\mathrm{MRCPCH}$

Tanuja Chitnis, MD

Daniela Pohl, MD, PhD

Maria Pia Amato, MD

Brenda Banwell, MD

Angelo Ghezzi, MD

Rogier Q. Hintzen, MD, $\mathrm{PhD}$

Lauren B. Krupp, MD

Naila Makhani, MD, $\mathrm{MPH}$

Kevin Rostásy, MD

Marc Tardieu, MD, PhD

Silvia Tenembaum, MD

Amy Waldman, MD

Emmanuelle Waubant, MD

Andrew J. Kornberg, MD

Correspondence to

E. Wassmer:

Evangeline.wassmer@bch.nhs.uk

\title{
International Pediatric MS Study Group Global Members Symposium report
}

\section{ABSTRACT}

The International Pediatric Multiple Sclerosis Study Group held its inaugural educational program, "The World of Pediatric MS: A Global Update," in September 2014 to discuss advances and challenges in the diagnosis and management of pediatric multiple sclerosis (MS) and other neuroinflammatory CNS disorders. Highlights included a discussion on the revised diagnostic criteria, which enable the differentiation of MS, acute disseminated encephalomyelitis, neuromyelitis optica, and other neuroinflammatory disorders. While these criteria currently identify clinical and MRI features for a particular diagnosis, advances in biomarkers may prove to be useful in the future. An update was also provided on environmental factors associated with pediatric MS risk and possibly outcomes, notably vitamin D deficiency. However, optimal vitamin D intake and its role in altering MS course in children have yet to be established. Regarding MS outcomes, our understanding of the cognitive consequences of early-onset MS has grown. However, further work is needed to define the course of cognitive function and its long-term outcome in diverse patient samples and to develop strategies for effective cognitive rehabilitation specifically tailored to children and adolescents. Finally, treatment strategies were discussed, including a need to consider additional drug treatment options and paradigms (escalation vs induction), although treatment should be tailored to the individual child. Of critical importance, clinical trials of newer MS agents in children are required. Although our understanding of childhood MS has improved, further research is needed to have a positive impact for children and their families. Neurology ${ }^{\circledR}$ 2016;87 (Suppl 2):S110-S116

\section{GLOSSARY}

ADEM = acute disseminated encephalomyelitis; $\mathbf{A D S}=$ acute demyelinating syndrome; $\mathbf{A Q P 4}=$ aquaporin- $\mathbf{C} \mathbf{C l}=$ confidence interval; $\mathbf{C I S}=$ clinically isolated syndrome; $\mathbf{D M T}=$ disease-modifying treatment; EDSS = Expanded Disability Status Scale; EMA = European Medicines Agency; FDA = Food and Drug Administration; IPMSSG = International Pediatric MS Study Group; $\mathbf{M O G}$ = myelin oligodendrocyte glycoprotein; $\mathbf{M S}=$ multiple sclerosis; $\mathbf{N M O}=$ neuromyelitis optica; NMOSD = neuromyelitis optica spectrum disorder; RDA = Recommended Dietary Allowance.

Advances in our understanding of epidemiology, pathogenesis, and treatment in multiple sclerosis (MS) have been rapid in the last few years and will have a positive effect for children with the disease. Such progress in the diagnosis and management of pediatric MS and neuroinflammatory disorders includes the following:

1. Revised diagnostic criteria for MS, acute disseminated encephalomyelitis (ADEM), and neuromyelitis optica (NMO) across the age spectrum.

2. Improved understanding of the cognitive consequences of MS onset during childhood.

3. Additional drug treatment options, including immunosuppressant, immunomodulatory, and oral medications, and awareness of the need for clinical trials of these agents in children.

\footnotetext{
From the Department of Neurology (E. Wassmer), Birmingham Children's Hospital, UK; Partners Pediatric MS Center (T.C.), Massachusetts General Hospital, Harvard Medical School, Boston; Department of Neurology (D.P.), Children's Hospital of Eastern Ontario, University of Ottawa, Canada; Department NEUROFARBA (M.P.A.), Section Neurosciences, University of Florence, Italy; Division of Neurology (A.W.), Perelman School of Medicine (B.B.), The Children's Hospital of Philadelphia, University of Pennsylvania; Divisione di Neurologia 2-Centro Studi Sclerosi Multipla (A.G.), Ospedale di Gallarate, Italy; Department of Neurology (R.Q.H.), MS Centre ErasMS, Erasmus MC, Rotterdam, the Netherlands; Lourie Center for Pediatric MS (L.B.K.), Stony Brook Children's, Stony Brook University, NY; Departments of Pediatrics and Neurology (N.M.), Yale University School of Medicine, New Haven, CT; Department of Pediatric Neurology (K.R.), Children's Hospital Datteln, University Witten/Herdecke, Germany; National Reference Center for Inflammatory Diseases of the Brain (M.T.), Hôpitaux Universitaires ParisSud, University Paris-Sud, France; Department of Neurology (S.T.), National Pediatric Hospital Dr. Juan P. Garrahan, Ciudad de Buenos Aires, Argentina; Pediatric MS Center (E. Waubant), UCSF Benioff Children's Hospital, and Neurology Department, UCSF, San Francisco, CA; and the Department of Neurology (A.J.K.), Royal Children's Hospital, Parkville, Australia.

Go to Neurology.org for full disclosures. Funding information and disclosures deemed relevant by the authors, if any, are provided at the end of the article.
} 
4. Consideration of environmental and dietary changes that may be of benefit to patients with MS, including vitamin D supplementation.

To discuss these areas of clinical and research development, challenges, and their effect upon children with MS, the International Pediatric MS Study Group (IPMSSG) held its inaugural educational program "The World of Pediatric MS: A Global Update.”

The IPMSSG has more than 200 members. The members are clinicians and researchers treating or studying children with MS. The MS International Federation sponsored the group. More information regarding the IPMSSG can be found on its Web site (www.ipmssg.org).

METHODS Participants. The meeting was held on September 9, 2014, in Boston, Massachusetts, funded by the US National MS Society, the Italian MS Society, and MS International Federation. The symposium brought together 72 IPMSSG members from 19 countries to learn from each other and share experience and expertise in the field of pediatric MS.

\section{Table Agenda of "The World of Pedatric MS: A Global Update"}

Am I missing something? Is it MS? Is it NMO?

MS and NMO diagnostic criteria

Prognostic markers in pediatric MS

Differential diagnosis

Role of brain biopsy

Role of myelin oligodendrocyte glycoprotein antibodies

"Vitamin D: To D or not to D?"

Vitamin D levels in the clinical context

Clinician's views of vitamin D supplementation (premeeting survey results)

Issues relating to monitoring and management strategies

Cognition: How can we help?

Cognitive issues in pediatric MS

Clinical and neuroimaging correlates

Contribution of fatigue and depression to cognitive impairment

Future research perspectives

MS treatments in 2 steps

Advantages and challenges of early disease-modifying treatment

Induction therapy, lessons learned in rheumatology

Role of MRI in therapeutic decisions

Defining inadequate treatment response

Clinical trials in pediatric MS and the IPMSSG response

Current and future trials

Challenges in clinical trials: Design, regulatory issues, treatment response, safety, and tolerability

MRI and cognition as future outcome measures

Abbreviations: IPMSSG = International Pediatric MS Study Group; MS = multiple sclerosis $\mathrm{NMO}=$ neuromyelitis optica.
Agenda. Five workgroups prepared 5 different topics, which were presented and discussed at the meeting (table).

DISCUSSION Diagnosis of MS and NMO. Diagnosis of MS has traditionally been made by clinical examination, using criteria supported by MRI. In children, the diagnostic criteria have only in recent years been defined and revised. ${ }^{1}$ ADEM and NMO need to be differentiated from MS. The recent advances in biomarkers (such as antibodies) may prove to be useful in diagnosis in the future.

The 2007 IPMSSG published consensus definitions for pediatric inflammatory demyelinating syndromes. ${ }^{2}$ In 2007, the main goal was to define common terminology with respect to the diagnosis for MS, ADEM, and NMO and establish a classification system to facilitate collaborative research studies. Some questioned the inclusion of encephalopathy in the criteria for ADEM. However, the presence of encephalopathy and polyfocal neurologic symptoms (ADEM presentation) in children younger than 12 years has been shown to accurately predict a monophasic course and low MS risk. ${ }^{3}$

In 2013, the IPMSSG revised the definitions, which included changes to the classification of relapses after ADEM. ${ }^{1}$ It was appreciated that other phenotypes of relapsing demyelinating disease occur such as ADEM followed by recurrent $\mathrm{ON}$ without other brain lesions (ADEM-ON) (as discussed in "Acute disseminated encephalomyelitis: Updates on an inflammatory CNS syndrome," p. S38). The revised IPMSSG definitions for MS enable an MS diagnosis at time of first attack, if baseline MRI findings satisfy dissemination in space and time criteria, as per the McDonald 2010 criteria for MS.

Children with MS diagnosed at the time of a first attack according to the 2010 criteria do not experience more frequent relapses ${ }^{4}$ and do not develop earlier physical disability when compared to patients with pediatric MS who did not meet criteria at the time of the first attack. $^{5}$ Prognostic factors for a more severe course include a shorter interval between 1 st and 2 nd MS attacks, low serum $25(\mathrm{OH})$ vitamin D level, ${ }^{6}$ and incomplete recovery from a severe 1 st attack. ${ }^{7,8}$

Data to predict response to treatment in pediatric patients with MS (e.g., frequency of relapses or MRI characteristics) are not yet available. In the future, the group agreed that it would be desirable to develop prognostic markers in order to individualize immunomodulatory treatment.

The IPMSSG consensus definitions include criteria for $\mathrm{NMO} .{ }^{1} \mathrm{NMO}$ criteria require that brain MRI characteristics are atypical for MS. ${ }^{9}$ The International Panel of NMO Diagnosis recommends that children and adults be allocated into 2 groups based on the aquaporin-4 (AQP4) antibody status measured with a cell-based assay: NMO spectrum disorder 
(NMOSD) with AQP4 antibodies or NMOSD without AQP4 antibodies. There are some differences between pediatric and adult NMO (as discussed in "Neuromyelitis optica spectrum disorders in children and adolescents," p. S59).

Pathology and diagnosis of demyelination. When typical clinical symptoms and consensus MRI criteria are met in pediatric patients with MS, a brain biopsy is not required to establish the diagnosis. Brain biopsy helps establish a diagnosis for children with an atypical severe acute demyelinating syndrome (ADS). ${ }^{10}$ The histopathologic results in children with clinically isolated syndrome (CIS) and MS are similar to findings in adults, characterized by an even more prominent perivascular and parenchymal inflammation with mainly $\mathrm{T}$ cells (CD8 > CD4), few B cells and plasma cells, in addition to an abundance of macrophages/microglia. Other important features include confluent demyelination with active signs of remyelination, acute axonal damage limited relative to the extent of demyelination, and activated astrocytes. Interestingly, children with NMO show inflammation with eosinophilic granulocytes, confluent demyelination, and axonal and oligodendrocyte loss. In addition, children with NMO and AQP4 antibodies reveal dystrophic astrocytes and loss of the protein AQP4 and upregulation of myelin oligodendrocyte glycoprotein (MOG) protein. One important difference in children with ADEM is that the inflammation is predominant and axons are relatively preserved.

Autoantibodies and diagnosis. Autoantibodies have recently gained attention because of the role of AQP4 antibodies in the diagnosis and pathogenesis of NMO. ${ }^{11}$ B-cells have long been implicated in various inflammatory-demyelinating diseases. Oligoclonal bands are a frequent finding in children with CIS/MS. Recently a number of articles were published examining the frequency of MOG antibodies in serum of children with $\mathrm{ADS} .^{12}$ MOG is expressed on the outermost surface of the myelin sheath. MOG antibodies induce complement mediated cytotoxicity in vitro. There is some preliminary evidence suggesting potential pathogenicity. ${ }^{13,14}$ Pooled data suggest that $25 \%$ of children with acute demyelination have MOG antibodies, and the presence of such anti-MOG antibodies occurs in ADEM, recurrent optic neuritis, in some children with AQP4-negative NMOSD, and less commonly in children experiencing the first attack of MS. ${ }^{15,16}$ The spectrum of MOGassociated diseases in childhood needs to be further defined and more research is required to determine diagnostic and therapeutic implications.

Cognition. Cognitive impairment is common in adults with MS. ${ }^{17}$ This has been studied less frequently in children. ${ }^{18-20}$ Cognitive impairment may differ in children as their burden of disease activity (clinical and MRI) is higher, but there is possibly better brain plasticity and ability for compensation in children. Research questions to be addressed are whether the development of MS during ongoing cerebral maturation leads to increased vulnerability of cognitive dysfunction or whether increased brain plasticity offers relative protection. Cognitive impairment in children with MS is estimated to occur in around $30 \%$ of the cases, especially in the young child. The pattern of cognitive dysfunction is similar to adults with deficits of information processing speed, memory, and executive functions. ${ }^{17}$ Different is the involvement of language with MS. Mild linguistic deficits can have great functional consequences especially with regards to academic achievements. The future study of large cohorts of patients with MS coupled with sensitive outcome measures and age-matched controls may help better define the nature and course of cognitive dysfunction in this age group and relative risk factors for varying developmental trajectories.

There may be a dissociation between physical and cognitive disability. ${ }^{21}$ Overall accrual of irreversible motor disability is slower in children than adults, but cognitive dysfunction can occur early in children, has been documented in CIS, and may progress in the absence of motor disability. The course of cognitive decline in MS has varied across different reports. ${ }^{22-25}$ Uniform sensitive assessment measures and longer periods of follow-up in large patient samples could clarify issues pertinent to rate of decline, relative risk factors, and effects on academic function. Psychosocial difficulties are frequent and contribute to cognitive dysfunction: fatigue is reported in $20 \%-50 \%$, depression varies from $6 \%$ to $30 \%,{ }^{26-30}$ and other psychiatric disorders such as anxiety can also occur. ${ }^{31}$ Fatigue and depression can impair the child's academic and daily functioning and quality of life.

Early identification of these cognitive and psychosocial issues is important so that these can be addressed at home and school. Whether early disease-modifying treatment (DMT) preserves cognitive function remains unclear. The development of cognitive rehabilitation techniques tailored to the needs of children is another major undertaking. Finally, MRI research in this field is in an early phase. ${ }^{32}$ Cognitive dysfunction has been associated with lesion load in the brain and corpus callosum and thalamic atrophy. Functional MRI studies may help understand underlying mechanisms and measure the effectiveness of cognitive rehabilitation.

Vitamin D and MS. In adult-onset MS, studies have shown that serum $25(\mathrm{OH}) \mathrm{D}$ levels are lower in patients with MS as compared to healthy controls ${ }^{33-35}$ 
and vitamin $\mathrm{D}$ levels appear to be lower during times of clinical relapse as compared to remission. ${ }^{34}$ Higher 25 $(\mathrm{OH}) \mathrm{D}$ levels have also been associated with reduced relapse risk, ${ }^{36,37}$ developing new or enhancing brain MRI lesions, and disability progression in adults with MS. ${ }^{38,39}$

A Canadian national prospective cohort study in pediatric patients with incident demyelination showed that a low serum $25(\mathrm{OH}) \mathrm{D}$ level at first presentation was associated with increased risk of subsequent MS diagnosis (hazard ratio [HR] 1.1, 95\% confidence interval [CI] $1.00-1.25$, for every 10 $\mathrm{nmol} / \mathrm{L}$ decrease). ${ }^{3}$ A US study suggested that relapse risk in pediatric patients with either MS or CIS was inversely related to serum $25(\mathrm{OH}) \mathrm{D}$ level with a $34 \%$ decrease in relapse risk for every $10 \mathrm{ng} / \mathrm{mL}(25 \mathrm{nmol} /$ $\mathrm{L}$ ) increase in serum vitamin $\mathrm{D}$ level (incidence risk ratio 0.66 , 95\% CI 0.46-0.95). ${ }^{6}$ These observations have led to ongoing clinical trials of vitamin D supplementation to confirm whether vitamin $\mathrm{D}$ changes the disease course.

The optimal amount of vitamin D intake for pediatric patients with MS remains unclear. The Recommended Dietary Allowance (RDA) for vitamin D suggested by the National Institute of Medicine is $600 \mathrm{IU} /$ day for all individuals over 1 year of age. This RDA was established to target a serum $25(\mathrm{OH}) \mathrm{D}$ level of $50 \mathrm{nmol} / \mathrm{L}$, which was determined with particular concern for maintaining bone health and preventing rickets, not for modulating immune function. Optimal vitamin D intake for improving MS course in children has yet to be established.

Overall, the group agreed that vitamin D3 doses between 600 and 4000 IU are likely safe. Establishing age-related oral vitamin $\mathrm{D}$ doses that are able to raise serum levels into the desired range, determining optimal frequency and timing of doses with respect to season, and establishing the safe range of supplemental oral vitamin $\mathrm{D}$ doses in children with $\mathrm{MS}$ will only occur through carefully designed prospective studies and randomized clinical trials.

Disease-modifying treatments. Current treatment options for first-line immunotherapy in relapsingremitting MS include interferon- $\beta$ and glatiramer acetate $^{40}$ (as discussed in "Pediatric multiple sclerosis: Conventional first-line treatment and general management"). However, some patients will experience breakthrough disease with these drugs. Escalation is a therapeutic strategy in which drugs with low risk are first utilized and, if needed, drugs with increasing toxicity are successively adopted..$^{41}$ The current concept of escalation therapy in MS involves switching patients who fail first-line therapy to more effective and riskier treatments (e.g., natalizumab), although these drugs have not been formally evaluated in children. Standardized algorithms and consensus definitions are required to monitor treatment outcome, to determine treatment failure, and to structure proceeding from one level of therapy to another.

A definition of inadequate treatment response is challenging. ${ }^{42}$ Current metrics used in adult MS include clinical relapses, MRI disease activity (new or enhancing or enlarging lesions), and accrual of irreversible disability (measured via Expanded Disability Status Scale [EDSS]). Considering that children rarely demonstrate sustained significant EDSS worsening, a treatment response algorithm combining clinical relapses and MRI findings appears reasonable. In view of the fact that treatment-naive pediatric patients with MS have an annualized relapse rate of 0.9-3.2 in the first 2 years, and that first-line DMTs should provide a reduction in relapses of $30 \%-40 \%$, an annual relapse rate of $>1$ may represent an insufficient treatment response. There is often marked MRI disease activity in children with MS and a mean of 9 new lesions in the first 6 months of follow-up has been described in a Canadian pediatric MS cohort. ${ }^{43}$ It is currently unclear how many new or contrastenhancing lesions should be considered as a marker for inadequate treatment response.

As in other autoimmune disorders, the group discussed that we may need to consider the concept of induction therapy at onset for some patients with highly active disease at risk of disability.

Induction represents an approach in which powerful immunosuppressant drugs are used from the beginning to treat the disease with objectives of stopping the disease activity early, resetting the immune system, and avoiding epitope spreading, preventing irreversible structural damage. An aggressive immunosuppressant should be considered for a limited time to gain control of disease, followed by maintenance therapy with lower risk drugs (e.g., glatiramer acetate or interferon- $\beta$ ) for patients with active disease and who are at risk for early accumulation of disability. This treatment strategy has been understudied and at this point has never been documented to work even in adult MS.

There was general agreement that treatment decisions should be tailored to the needs and status of the child and taken after discussion with the young person and his or her family.

Treatment trials. There is a lack of evidence on the use of DMTs in children with MS. ${ }^{40}$ The US Food and Drug Administration (FDA) has not approved any therapies for pediatric MS. The European Medicines Agency (EMA) has approved limited use of interferon in adolescents with MS. Around $40 \%$ of children with MS discontinue treatment due to medication 
intolerance, side effects, or relapses. There is a need for novel treatments. Several new drugs have shown efficacy in phase III trials and have been approved or will be for adults with MS. Both EMA and FDA mandate pediatric studies for new medicinal products. A waiver is possible when it is not appropriate to develop certain medicines in children: the disorder only occurs in adulthood, a study is not feasible, or the treatment is unsafe or ineffective in childhood. In the United States, the Best Pharmaceuticals Act for Children provides for voluntary pediatric drug assessments and the FDA is able to issue written requests for pediatric studies. The incentive for industry to conduct these studies is an additional 6 months of market exclusivity.

Several challenges were identified for clinical trials in pediatric MS, including the small number of children, as this is a rare disorder. Ideally, one trial design should be used for all countries. Study designs considered are randomized controlled trials with an active comparator or placebo. Placebo will reduce number of patients needed to power the study, but is unacceptable to some regulatory agencies, patients, and clinicians, especially if the study duration is long. ${ }^{44}$ The outcome measures used in adult trials are not necessarily sensitive in measuring disability in children. The rigor and intensity with frequent clinic visits and blood samples make the implementation of studies difficult. Strategies to increase knowledge about clinical trial conduct among pediatric MS practitioners were discussed.

There has been progress in our understanding of childhood MS and neuroinflammatory disorders; however, further research is required to understand the diagnostic and therapeutic implications of these advances.

The revised definitions and diagnostic criteria enable ADEM and NMO to be differentiated from MS. MS diagnosis is now possible at time of first attack, if MRI findings satisfy McDonald 2010 criteria. MRI features aid in the differentiation of MS, ADEM, and NMO. Serum antibodies directed against AQP4 provide diagnostic support for NMO, while the clinical and therapeutic importance of antibodies against MOG remains to be clarified.

Cognitive impairment, depression, and fatigue are all important facets of MS in children, and development of rehabilitative and therapeutic strategies for cognitive impairment and fatigue, and identification and care of emotional issues, are relevant for all pediatric patients with MS. Clinical trials of vitamin D supplementation are required to better determine whether vitamin $\mathrm{D}$ supplementation influences disease activity and at what doses.

Current treatments for first-line immunotherapy in paediatric MS include interferon- $\beta$ and glatiramer acetate. Escalation strategies may be beneficial for children with inadequate treatment response. For some children with an aggressive disease evolution, induction therapy may need to be considered.

There is limited evidence of DMTs in children with MS. Both Europe and the United States mandate pediatric studies for new medicinal products. Although several challenges for clinical trials in pediatric MS have been identified, there are strategies being implemented to address these, and there is a strong desire within the pediatric MS community and the IPMSSG for further research.

\section{AUTHOR CONTRIBUTIONS}

All authors contributed to acquisition and interpretation of data (literature review), writing, and critical revision of the manuscript.

\section{ACKNOWLEDGMENT}

The authors thank all the participants at the meeting, who contributed to the discussions and consensus statements; and the following colleagues, who searched the literature and prepared, reviewed, and edited the intellectual content of the workgroups: Diagnosis Workgroup: Dr. Wolfgang Brück, Dr. Manikum Moodley, Dr. Jayne Ness, Dr. Daniela Pohl, Dr. Marc Tardieu, Dr. Silvia Tenembaum, Dr. Emmanuelle Waubant, Dr. Amy Waldmann, Dr. Kevin Rostasy. Cognition Workgroup: Dr. Maria Pia Amato, Dr. Lauren Krupp, Dr. Joy Parrish, Dr. Leigh Charvet, Dr. E. Portaccio, Dr. Michael Absoud, Dr. Iris Penner, Dr. Shay Menascu, Dr. Bianca Weinstock-Guttmann. Vit D Workgroup: Dr. Brenda Banwell, Dr. Heather Hanwell, Dr. Rogier Hintzen, Dr. Joaquin Pena, Dr. Anne-Louise Ponsonby, Dr. Sunita Venkateswaran, Dr. Dessa Sadovnick, Dr. Alberto Ascherio, Dr. Naila Makhani. Treatment Workgroup: Dr. Daniela Pohl, Dr. Barbara Bajer-Kornek, Dr. Angelo Ghezzi, Dr. Cheryl Hemingway, Dr. Peter Huppke, Dr. Evangeline Wassmer, Dr. Michael Kruer, Dr. Francesco Patti. Clinical Trials Workgroup: Dr. Marc Tardieu, Dr. Brenda Banwell, Dr. Tanuja Chitnis, Dr. Kumuran Deiva, Dr. Andrew Kornberg, Dr. Mary Rensel, Dr. Amy Waldman.

\section{STUDY FUNDING}

This supplement is made possible by funding from the MS Cure Fund, Danish MS Society, German MS Society, Italian MS Association, MS International Federation, MS Research Foundation (Netherlands), National MS Society (USA) and Swiss MS Society.

\section{DISCLOSURE}

E. Wassmer received honoraria for speaking/travel costs from Biogen Idec, Teva, Genzyme, Shire, UCB, and Merck Serono. She is an investigator in trials with Biogen Idec, Sanofi, and Novartis. Her MS research projects have been funded by the UK MS Society, Action Medical Research, and Birmingham Children's Hospital Research Foundation. T. Chitnis has received personal compensation for advisory board/consulting for Biogen Idec, Merck-Serono, and Novartis, and has received research support from Merck-Serono and Novartis Pharmaceuticals. She serves on clinical trial advisory boards for Genzyme-Sanofi and Novartis. D. Pohl reports no disclosures relevant to the manuscript. M. Amato has received research grants and honoraria as a speaker and member of advisory boards by Bayer, Biogen Idec, Merck Serono, Novartis, Sanofi Genzyme, Teva, and Almirall. B. Banwell serves as an unpaid consultant for Novartis, Biogen-Idec, Sanofi, and Teva Neuroscience. She serves as a central MRI reviewer for a clinical trial sponsored by Novartis. A. Ghezzi received honoraria for speaking from Biogen Idec, MerckSerono, Novartis, Genzyme, Teva, and Allergan; for consultancy from Merck-Serono, Teva, Novartis, and Biogen Idec; and received support for participation in national and international congresses from Schering, Biogen Idec, Merck-Serono, Novartis, Genzyme, and Teva. R. Hintzen received honoraria for serving on advisory boards for Biogen Idec, Roche, and Sanofi. He participated in trials with Biogen Idec, Merck-Serono, 
Roche, Genzyme, and Novartis. L. Krupp received honoraria or consultation fees from Biogen, Novartis, Teva Neurosciences, Pfizer, Projects in Knowledge, Providence Health Services, and Multicell, and royalty payments from Avanir Pharmaceuticals, Abbvie Inc., Osmotica Pharmaceutical Corp., and Demos Medical Publishing; and received research funding from Novartis, Teva Neurosciences, Biogen Idec, Genentech, National Multiple Sclerosis Society, Department of Defense, National Institutes of Health, and the Lourie Foundation. N. Makhani received fellowship funding from the Canadian Network of MS Clinics and has received research funding from the Dairy Farmers of Ontario and Race to Erase MS. She is a site investigator for a clinical trial sponsored by Novartis. K. Rostasy has served on an advisory board for a pediatric MS trial with Novartis. M. Tardieu reports no disclosures relevant to the manuscript. S. Tenembaum served as an advisory board member or speaker for Merck Serono. She serves on a clinical trial advisory board for Genzyme-Sanofi. Professional travel/accommodations expenses have been awarded to her by Merck-Serono. A. Waldman receives funding from the NIH (NINDS K23), NMSS, Novartis, and Biogen. E. Waubant received funding from grants from the NIH, National MS Society, Race to Erase MS, and one educational lecture (Genentech); is an investigator in trials with Roche, Novartis, and Biogen; and is on the advisory board of a Novartis trial (volunteer). A. Kornberg is PI on 2 pediatric MS trials sponsored by Novartis and Sanofi. He has had travel support for educational meetings as a speaker by CSL Bioplasma, Novartis, and Biogen Idec. He has served on an advisory board for pediatric MS trials with Biogen Idec. Go to Neurology.org for full disclosures.

Received August 19, 2015. Accepted in final form March 28, 2016.

\section{REFERENCES}

1. Krupp LB, Tardieu M, Amato MP, et al; International Pediatric Multiple Sclerosis Study Group. International Pediatric Multiple Sclerosis Study Group criteria for pediatric multiple sclerosis and immune-mediated central nervous system demyelinating disorders: revisions to the 2007 definitions. Mult Scler 2013;19:1261-1267.

2. Krupp LB, Banwell B, Tenembaum S; International Pediatric MS Study Group. Consensus definitions proposed for pediatric multiple sclerosis and related disorders. Neurology 2007;68(16 suppl 2):S7-S12.

3. Banwell B, Bar-Or A, Arnold DL, et al; Clinical, environmental, and genetic determinants of multiple sclerosis in children with acute demyelination: a prospective national cohort study. Lancet Neurol 2011;10:436-453.

4. van Pelt ED, Neuteboom RF, Ketelslegers IA, Boon M, Catsman-Berrevoets CE, Hintzen RQ; Dutch Study Group for Paediatric MS. Application of the 2012 revised diagnostic definitions for paediatric multiple sclerosis and immunemediated central nervous system demyelination disorders. J Neurol Neurosurg Psychiatry 2014;85:790-794.

5. Bigi S, Marrie RA, Verhey L, Yeh EA, Banwell B. 2010 McDonald criteria in a pediatric cohort: is positivity at onset associated with a more aggressive multiple sclerosis course? Mult Scler 2013;19:1359-1362.

6. Mowry E, Krupp L, Milazzo M, et al. Vitamin D status is associated with relapse rate in pediatric-onset multiple sclerosis. Ann Neurol 2010;67:618-624.

7. Fay AJ, Mowry EM, Strober J, Waubant E. Relapse severity and recovery in early pediatric MS. Mult Scler 2012; 18:1008-1012.

8. Harding KE, Liang K, Cossburn MD, et al. Long-term outcome of paediatric-onset multiple sclerosis: a population-based study. J Neurol Neurosurg Psychiatry 2013;84: 141-147.

9. Kim HJ, Paul F, Lana-Peixoto MA, et al; Guthy-Jackson Charitable Foundation NMO International Clinical Consortium \& Biorepository. MRI characteristics of neuromyelitis optica spectrum disorder: an international update. Neurology 2015;84:1165-1173.

10. Kuhlmann T, Lassmann H, Brück W. Diagnosis of inflammatory demyelination in biopsy specimens: a practical approach. Acta Neuropathol 2008;115:275-287.

11. Hacohen Y, Absoud M, Woodhall M, et al; UK \& Ireland Childhood CNS Inflammatory Demyelination Working Group. Autoantibody biomarkers in childhood-acquired demyelinating syndromes: results from a national surveillance cohort. J Neurol Neurosurg Psychiatry 2014;85:456-461.

12. Rostasy K, Reindl M. Role of autoantibodies in acquired inflammatory demyelinating diseases of the central nervous system in children. Neuropediatrics 2013;44:297-301.

13. Dale RC, Tantsis EM, Merheb V, et al. Antibodies to MOG have a demyelination phenotype and affect oligodendrocyte cytoskeleton. Neurol Neuroimmunol Neuroinflamm 2014;1:e12.

14. Spadaro M, Gerdes LA, Mayer MC, et al. Histopathology and clinical course of MOG-antibody-associated encephalomyelitis. Ann Clin Transl Neurol 2015;2:295-301.

15. Hacohen Y, Absoud M, Deiva K, et al. Myelin oligodendrocyte glycoprotein antibodies are associated with a non-MS course in children. Neurol Neuroimmunol Neuroinflamm 2015;2:e81.

16. Ketelslegers IA, Van Pelt DE, Bryde S, et al. Anti-MOG antibodies plead against MS diagnosis in an acquired demyelinating syndromes cohort. Mult Scler 2015;21: 1513-1520.

17. Blaschek A, Storm van's Gravesande K, Heinen F, et al. Neuropsychological aspects of childhood multiple sclerosis: an overview. Neuropediatrics 2012;43:176-183.

18. Amato MP, Goretti B, Ghezzi A, et al; Multiple Sclerosis Study Group of the Italian Neurological Society. Cognitive and psychosocial features of childhood and juvenile MS. Neurology 2008;70:1891-1897.

19. Julian L, Serafin D, Charvet L, et al; Network of Pediatric MS Centers of Excellence. Cognitive impairment occurs in children and adolescents with multiple sclerosis: results from a United States network. J Child Neurol 2013;28: 102-107.

20. MacAllister WS, Belman A, Milazzo M, et al. Cognitive functioning in children and adolescents with multiple sclerosis. Neurology 2005;64:1422-1425.

21. Yeh EA, Parrish JB, Weinstock-Guttman B. Disease progression in pediatric multiple sclerosis: disparities between physical and neurocognitive outcomes. Expert Rev Neurother 2011;11:433-440.

22. Amato MP, Goretti B, Ghezzi A, et al; Multiple Sclerosis Study Group of the Italian Neurological Society. Cognitive and psychosocial features in childhood and juvenile MS: two-year follow-up. Neurology 2010;75:1134-1140.

23. Charvet LE, O'Donnell EH, Belman AL, et al. Longitudinal evaluation of cognitive functioning in pediatric multiple sclerosis: report from the US Pediatric Multiple Sclerosis Network. Mult Scler 2014;20:1502-1510.

24. MacAllister WS, Christodoulou C, Milazzo M, Krupp L. Longitudinal neuropsychological assessment in pediatric multiple sclerosis. Dev Neuropsychol 2007;32:625-644.

25. Till C, Racine N, Araujo D, et al. Changes in cognitive performance over a 1-year period in children and adolescents with multiple sclerosis. Neuropsychology 2013;27: 210-219.

26. Goretti B, Portaccio E, Ghezzi A, et al; Multiple Sclerosis Study Group of the Italian Neurological Society. Fatigue 
and its relationships with cognitive functioning and depression in paediatric multiple sclerosis. Mult Scler 2012;18:329-334.

27. Holland AA, Graves D, Greenberg BM, Harder LL. Fatigue, emotional functioning, and executive dysfunction in pediatric multiple sclerosis. Child Neuropsychol 2014;20:71-85.

28. MacAllister WS, Christodoulou C, Troxell R, et al. Fatigue and quality of life in pediatric multiple sclerosis. Mult Scler 2009;15:1502-1508.

29. Parrish JB, Weinstock-Guttman B, Smerbeck A, Benedict RH, Yeh EA. Fatigue and depression in children with demyelinating disorders. J Child Neurol 2013;28:713-718.

30. Till C, Udler E, Ghassemi R, et al. Factors associated with emotional and behavioral outcomes in adolescents with multiple sclerosis. Mult Scler 2012;18:1170-1180.

31. Weisbrot D, Charvet L, Serafin D, et al. Psychiatric diagnoses and cognitive impairment in pediatric multiple sclerosis. Mult Scler 2014;20:588-593.

32. Till C, Ghassemi R, Aubert-Broche B, et al. MRI correlates of cognitive impairment in childhood-onset multiple sclerosis. Neuropsychology 2011;25:319-332.

33. van der Mei IA, Ponsonby AL, Dwyer T, et al. Vitamin D levels in people with multiple sclerosis and community controls in Tasmania, Australia. J Neurol 2007;254:581-590.

34. Kragt J, van Amerongen B, Killestein J, et al. Higher levels of 25-hydroxyvitamin D are associated with a lower incidence of multiple sclerosis only in women. Mult Scler 2009;15:9-15.

35. Soilu-Hanninen M, Airas L, Mononen I, Heikkila A, Viljanen M, Hanninen A. 25-Hydroxyvitamin D levels in serum at the onset of multiple sclerosis. Mult Scler 2005;11:266-271.
36. Simpson S Jr, Taylor B, Blizzard L, et al. Higher 25-hydroxyvitamin $\mathrm{D}$ is associated with lower relapse risk in multiple sclerosis. Ann Neurol 2010;68:193-203.

37. Runia TF, Hop WC, de Rijke YB, Buljevac D, Hintzen RQ. Lower serum vitamin D levels are associated with a higher relapse risk in multiple sclerosis. Neurology 2012;79:261-266.

38. Ascherio A, Munger KL, White R, et al. Vitamin D as an early predictor of multiple sclerosis activity and progression. JAMA Neurol 2014;71:306-314.

39. Mowry EM, Waubant E, McCulloch CE, et al. Vitamin D status predicts new brain magnetic resonance imaging activity in multiple sclerosis. Ann Neurol 2012;72: 234-240

40. Chitnis T, Tenembaum S, Banwell B, et al; International Pediatric Multiple Sclerosis Study Group. Consensus statement: evaluation of new and existing therapeutics for pediatric multiple sclerosis. Mult Scler 2012;18:116-127.

41. Rieckmann P. Concepts of induction and escalation therapy in multiple sclerosis. J Neurol Sci 2009;277(suppl 1): S42-S45.

42. Yeh EA, Waubant E, Krupp LB, et al; National Network of Pediatric MS Centers of Excellence. Multiple sclerosis therapies in pediatric patients with refractory multiple sclerosis. Arch Neurol 2011;68:437-444.

43. Yeh EA, Weinstock-Guttman B, Ramanathan M, et al. Magnetic resonance imaging characteristics of children and adults with paediatric-onset multiple sclerosis. Brain 2009;132:3392-3400.

44. Chitnis T, Tardieu M, Amato MP, et al. International Pediatric MS Study Group Clinical Trials Summit: meeting report. Neurology 2013;80:1161-1168. 


\section{Neurology}

International Pediatric MS Study Group Global Members Symposium report Evangeline Wassmer, Tanuja Chitnis, Daniela Pohl, et al.

Neurology 2016;87;S110-S116

DOI 10.1212/WNL.0000000000002880

This information is current as of August 29, 2016

Updated Information \&

Services

References

Subspecialty Collections

Permissions \& Licensing

Reprints including high resolution figures, can be found at: http://n.neurology.org/content/87/9_Supplement_2/S110.full

This article cites 44 articles, 3 of which you can access for free at: http://n.neurology.org/content/87/9_Supplement_2/S110.full\#ref-list-1

This article, along with others on similar topics, appears in the following collection(s):

Adolescence

http://n.neurology.org/cgi/collection/adolescence All Demyelinating disease (CNS)

http://n.neurology.org/cgi/collection/all_demyelinating_disease_cns All Pediatric

http://n.neurology.org/cgi/collection/all_pediatric

Multiple sclerosis

http://n.neurology.org/cgi/collection/multiple_sclerosis

Information about reproducing this article in parts (figures,tables) or in its entirety can be found online at:

http://www.neurology.org/about/about_the_journal\#permissions

Information about ordering reprints can be found online:

http://n.neurology.org/subscribers/advertise

Neurology ${ }^{\circledR}$ is the official journal of the American Academy of Neurology. Published continuously since 1951, it is now a weekly with 48 issues per year. Copyright @ 2016 American Academy of Neurology. All rights reserved. Print ISSN: 0028-3878. Online ISSN: 1526-632X.

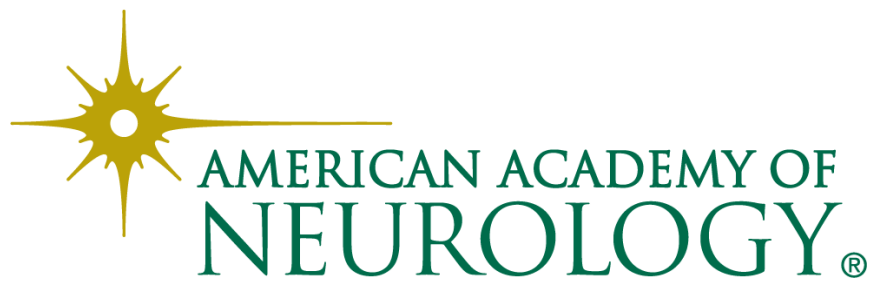

